\title{
Acerca de las creencias, actitudes y visión ecológica del mundo en estudiantes chilenos. Un estudio preliminar enmarcado en la Educación para la Sustentabilidad
}

\section{Relative to the beliefs, attitudes and ecologic vision of the world in Chilean students. A preliminary study framed in the concept of Education for Sustainability}

\author{
Enrique Zambra Araneda \\ Ana M. Cáceres Palavecino ${ }^{1}$ \\ Universidad Santo Tomás de Chile- Talca \\ Susanne Menzel \\ Universidad de Gottingen, Alemania
}

(Recepción: Mayo 2006- Aceptación: Octubre 2006)

\begin{abstract}
La Organización de las Naciones Unidas ha realizado un interesante llamado a la comunidad mundial para la celebración del decenio 2005-2014, declarado como "Década de la educación para el desarrollo sostenible". Este llamado surge ante la evidencia de que hoy en día vivimos una situación de auténtica emergencia planetaria: contaminación y degradación de los ecosistemas, crecimiento demográfico desmedido, sobreexplotación de recursos, desequilibrios insostenibles, pérdida de la diversidad cultural y biológica, ctc.

En Chile, específicamente, ya poseemos un documento borrador $\mathrm{N}^{\mathrm{a}} 4$ que data del 31 de enero de 2006 sobre Política Nacional de Educación para el Desarrollo Sustentable, donde se destaca la educación como un instrumento de gestión ambiental, además de los principios y objetivos sobresalientes que permitirán la emergencia de una nueva visión de nuestro mundo. Se trata entonces de generar en las comunidades escolares y ciudadana un compromiso por una educación para la sustentabilidad donde jueguen un papel significativo los valores, creencias, actitudes y comportamientos a nivel individual y social.
\end{abstract}

Palabras clave: Desarrollo sustentable, educación, actitudes

\begin{abstract}
United Nations has made an interesting call to the world community for the celebration of the decade from 2005 to 2014 declared as "The decade of education for sustainable development" this call comes foreword because of the evidence that today we are living a situation of real planetary emergency: Pollution and degradation of ecosystems, population increasing rapidly and without any control, overexplotation of resources, unsustainable unbalances, losses in the cultural and biological diversity, etc.

In Chile, specifically, we already possess a rough draft $\mathrm{N}^{\mathrm{a}} 4$ dated 31 of January of 2006 of National Education Policy for Sustainable Development, where education is emphasized as an instrument of ambiental management, besides the outstanding principles and objectives that will permit the creation of a new vision of our world. It is about generating in educational and civic communitics a commitment for an education for the sustainability where the values, beliefs, attitudes and behaviors plays a significant role at individual and social level.
\end{abstract}

Keywords: Sustainable Development, Education, Attitudes.

La Organización de las Naciones Unidas ha realizado un interesante llamado a la comunidad mundial para la celebración del decenio 2005-2014, declarado como "Década de la educación para el desarrollo sostenible". Este llamado surge ante la evidencia de que hoy en día vivimos una situación de auténtica emergencia planetaria: contaminación y degradación de los

1 Correspondencia a: Ana M. Cácercs Palavecino. E mail: acaceres@santotomas.cl ecosistemas, crecimiento demográfico desmedido, sobreexplotación de recursos, desequilibrios insostenibles, pérdida de la diversidad cultural y biológica, etc.

Se hace necesario entonces, recurrir a la formación de ciudadanas y ciudadanos conscientes de la gravedad y del carácter global de los problemas y prepararlos para participar en la toma de decisiones adecuadas. En Chile, específicamente, ya poseemos un documento borra- 
dor $\mathrm{N}^{\circ} 4$ que data del 31 de enero de 2006 sobre Política Nacional de Educación para el Desarrollo Sustentable, donde se destaca la educación como un instrumento de gestión ambiental, además de los principios y objetivos sobresalientes que permitirán la emergencia de una nueva visión de nuestro mundo.

Se trata, entonces de generar en las comunidades escolares y ciudadana un compromiso por una educación para la sustentabilidad donde jueguen un papel significativo los valores, creencias, actitudes y comportamientos a nivel individual y social.

\section{Valores, creencias, actitudes y comportamiento ambiental}

El término actitud se introduce en la psicología social en la década del 20 del siglo pasado en los Estados Unidos, debido a la ocurrencia de cambios sociales y políticos en esta sociedad, que originaron un marcado interés en el análisis de los mecanismos reguladores del comportamiento humano. Desde entonces se han desarrollado numerosas investigaciones con diferentes enfoques acerca de este complejo y multifacético proceso psicológico de gran importancia en la educación de la población.

Según Allport (1935), citado por Casales), la actitud consiste en "un estado mental y neural de disposición, organizado a través de la experiencia, que ejerceria una influencia directiva o dinámica sobre la conducta del individuo ante todos los objetos y situaciones con los que se relaciona".

Casales (1989) señala varias características esenciales de las actitudes:

1. Son estructuras psicológicas, procesos derivados del aprendizaje y la experiencia del individuo, que constituyen el resultado de su actividad. Es decir, las actitudes se forman y desarrollan a lo largo de toda la vida del individuo, condicionadas por el contexto social al cual están expuestos, en dependencia de los grupos a los que pertenecen, los cuales se estructuran de acuerdo con el sistema de valores imperantes en la sociedad.

2. Aunque son el resultado de la actividad del individuo, son modificadas por esta; las actitudes condicionan y modifican las actividades subyacentes.
3. Tienen la propiedad de reflejarse en el comportamiento del individuo, de orientarlo, de regularlo y guiarlo en determinado sentido.

4. En toda actitud puede distinguirse su objeto, dirección e intensidad.

Por otro lado, a partir de investigaciones más recientes, se ha demostrado que las relaciones entre actitudes y comportamiento no son siempre lineales, por lo cual no podemos interpretarlas de manera simplista y mecánica. En otras palabras, la actitudes no cumplen una función predictiva de la conducta humana. El hecho de que ésta constituya una disposición a la reacción, no significa que siempre va a ser correspondiente con ella, ya que las mismas no constituyen el único factor determinante del comportamiento humano, en el cual, además de las actitudes, pueden influir la situación específica en la que se encuentra el sujeto a la hora de actuar, sus valores intrínsecos, el aprendizaje de conductas, normas grupales, características individuales o las consecuencias previsibles de la conducta.

No obstante, es innegable que las actitudes y creencias juegan un papel importante y fundamental en la toma de decisiones y en las acciones concretas de los seres humanos. Es por ello que el estudio de las actitudes, creencias específicas y percepción o visión del mundo, han sido preocupación de muchos psicólogos sociales y de la educación, desde que comienza el análisis más crítico y profundo de los intereses, comportamientos, creencias y valores que presentan los individuos frente a diversas situaciones, donde hoy en día destaca el comportamiento frente al medio ambiente, sobre todo de nuestros educandos.

En el contexto ambiental, para Febles (1999), las actitudes ambientales deben definirse como una "predisposición del pensamiento humano a actuar a favor o en contra del entorno social, teniendo como base las vivencias, los conocimientos y los valores del individuo con respecto a su entorno".

Es así como las actitudes ambientales cumplen con funciones tales como:

1. Selección de ambientes. Ayuda a seleccionar el ambiente donde se desea vivir, trabajar, estudiar o divertirse. Dicha elección conlleva un proceso de reflexión, análisis y predicción de futuras consecuencias de su comportamiento. Esta elección estará determi- 
nada por el nivel de satisfacción-insatisfacción de las necesidades individuales en dicho espacio.

2. Protección del entorno natural. Las actitudes ambientales también ayudan a los individuos en la toma de decisiones y en el uso y preservación del medio ambiente.

Stern y colaboradores (1995), enfatizan que la estructura social actúa de dos formas frente a la manifestación de las conductas humanas. Estaría moldeando las primeras experiencias y, en consecuencia, los valores del individuo y las creencias generales o visión del mundo. Aquella también incidiría en proporcionar fuerza y oportunidades para dar forma al comportamiento.

Los autores citados en el párrafo anterior, consideran a los valores, creencias generales y visón del mundo como antecedentes causales de creencias más específicas (ver figura 1), las cuales a su vez, son antecedentes para mantener normas e intenciones de acciones particulares. Los valores y percepción del mundo actuarían como filtros de nuevas ideas o de informaciones, considerando que ellos se generan o forman muy precozmente en la vida del individuo dentro de la familia; mientras que las creencias o formas de ver nuestro entorno, pueden ser el resultado de experiencias sociales y políticas más allá del entorno familiar.

Los valores, probablemente, son más estables en el curso de la vida, ya que ellos pueden ser desafiados individualmente en términos de su conveniencia, pero no en el contexto de su veracidad.

Fig. 1. Modelo causal esquemático en el contexto ambiental

Posición en la estructura social

Valores

Creencias Generales y visión del mundo

Creencias específicas y actitudes específicas

Compromisos conductuales o intenciones

Comportamiento o conducta

\section{Comportamiento ambiental}

Bajo este concepto se pueden agrupar las diferentes acciones del sujeto, dirigidas a modificar aspectos del entorno o de la relación con el mismo, y que influyen a su vez en las concepciones, percepciones y sensibilidades que posee la persona sobre el medio. Esto puede darse en dos sentidos: positivo y negativo, dependiendo del grado de compromiso del sujeto con su medio ambiente.

A pesar de que el conocimiento, las percepciones, actitudes, sensibilidades, valores y comportamientos ambientales se han analizado mucho desde la psicología, los resultados de numerosos estudios de las relaciones entre estos procesos son aparentemente contradictorios, sugiriendo, según Nieto (2003), ciertas interrogantes: ¿cómo se convierten el conocimiento y la preocupación ambiental en conductas y acciones pro ambientalistas? ¿Por qué pueden las personas estar supuestamente preocupados, tener conocimientos adecuados sobre medio ambiente, sin que esto se traduzca necesariamente en comportamientos a favor del mismo? ¿En qué consiste la brecha existente entre pensamientos, emociones, actitudes, percepciones con respecto a la conducta del individuo?

En este mismo rumbo, Stern y colaboradores (1999) desarrollaron una teoría compleja denominada teoría VBN (valor-creencia-norma). Esta propuesta (Fig.2) logra ligar la teoría de valores con aquella conocida como activadora de la norma y la perspectiva del nuevo paradigma ecológico (NEP). En otras palabras, esta nueva teoría unifica: los valores personales (especialmente los valores altruistas), la visión ecológica del mundo (NEP), la creencia en estar consciente de las consecuencias adversas (AC) de una práctica no amigable con el medio ambiente, y qué acciones cree una persona podría llegar a iniciar para evitar dichas consecuencias al atribuirse responsabilidad (AR) y así reducir la amenaza. 
Fig. 1. Representación esquemática de variables en la Teoría VBN (según Stern, 2000). Las flechas representan efectos directos*

Valores

Creencias

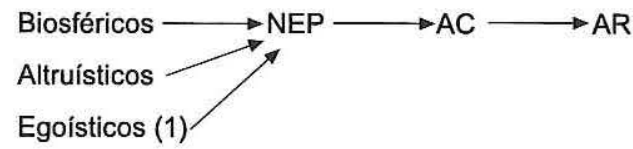

Es evidente que entre los procesos psicológicos, en el contexto ambiental, no existe una relación automática, mecánica o directa, es decir, entre conocimiento, conciencia, sensibilización y preocupación ambiental por una parte, y conductas o comportamientos ambientales, por otro; los mismos se relacionan de manera dialéctica y no existen independientemente unos de otros, sino que se encuentran interrelacionados genética, estructural y funcionalmente; ellos van formando un complejo sistema de motivos orientados a la regulación de la relación entre el individuo y su entorno.

El presente trabajo delimita su acción en el contexto de las creencias generales o primitivas acerca de las relaciones hombre-ambiente, basándose la adquisición de datos en un cuestionario denominado "Nuevo Paradigma Ecológico" NEP (New Ecological Paradigm), el cual es considerado un armazón o esqueleto conceptual que incorpora un trabajo socio-psicológico sobre los asuntos ambientales. A su vez muchos investigadores piensan que los ítems de la escala NEP fundamenta creencias y actitudes ambientales más específicas.

Ellos utilizan el término paradigma para sugerir que el NEP representa una "nueva perspectiva revolucionaria"; una estructura cognitiva coherente o visión del mundo. La escala pre-

\footnotetext{
* Esta teoría de Stern y colaboradores (1999) utiliza aspectos de la Teoría altruista de la activación de la norma moral. Esta tcoría generada por Schwartz $(1970,1977)$ sostiene que el comportamiento altruista, incluyendo el proambiental, se presenta en respuesta a normas morales personales que son activadas en individuos que poseen la creencia de que condiciones particulares ofrecen amenazas a otras, es decir, poseen la sensibilidad para captar las consecuencias adversas (AC) y que las acciones que ellos pueden emprender, podrían evitar consecuencias atribuyéndose responsabilidad (AR).
}

Normas Personales

Proambientalistas

Comportamiento

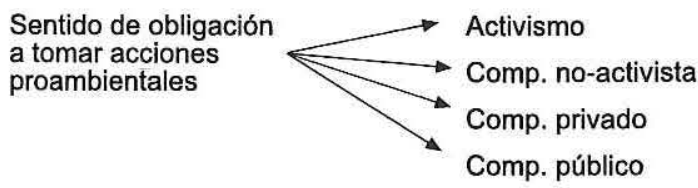

tende medir un tipo de teoría ecológica popular de cómo trabaja el mundo, la naturaleza de la biosfera, cómo ella funciona y se ve afectada por las acciones humanas. Observando las figuras 1 y 2 es posible descubrir dónde se adapta, dentro de una teoría o modelo de interés ambiental, tal conjunto de creencias generales.

NEP es una encuesta tipo Likert con 15 ítems. Cada faceta hipotética de visón ecológica del mundo posee tres (3) ítems: la realidad o concepción de poner límites al crecimiento (1, $6,11)$, antropocentrismo $(2,7,12)$, la fragilidad del equilibrio de la naturaleza $(3,8,13)$, rechazo a la idea de una "humanidad exenta o inmune", es decir, de que los humanos - a diferencia de otras especies- está exentos de las fuerzas de la naturaleza $(4,9,14)$ y la posibilidad de una ecocrisis $(5,10,15)$. Cada ítem presenta cinco opciones de respuesta: Totalmente de Acuerdo (TA), Parcialmente de acuerdo (PA), Inseguridad o Neutralidad (I) en su respuesta, Casi en Desacuerdo (CD) y Totalmente en Desacuerdo (TD).

El objetivo del presente trabajo es medir y analizar en forma incipiente, las creencias y actitudes que presentan los estudiantes chilenos del grupo etario 14-16 años, con respecto a los fenómenos de naturaleza ambiental de gran incidencia para el futuro de la humanidad.

Para cumplir con dicho objetivo, un total de 356 estudiantes (137 hombres y 119 mujeres), de Octavo Básico a Segundo Medio, de diversas regiones del país y con diferencial dependencia de establecimientos educacionales (municipales, subvencionados y particulares pagados), fueron sometidos a la encuesta NEP mientras participaban en una Feria Científica-Tecnológica Estudiantil llevada a cabo en el Museo 
Nacional de Historia Natural en Santiago de Chile durante el mes de octubre del año 2005. La muestra fue elegida aleatoriamente de entre los miles de estudiantes asistentes a dicho evento. Metodológicamente hablando, los integrantes de la muestra no se obtuvieron proporcionalmente según el establecimiento del cual provienen. Esto significa que en la muestra analizada había estudiantes de diferentes niveles socioeconómicos, no necesariamente en proporción directa y representativa del universo de tipos de establecimientos educacionales del país.

Al no existir dentro de la bibliografía consultada una versión en español de dicha encuesta, se procedió a su traducción desde el idioma inglés, respetando al máximo la intención original del ítem correspondiente y claridad en la sintaxis para la comprensión del ítem por parte del encuestado.

Las Tablas 1 y 2 muestran las distribuciones porcentuales para cada uno de los items separadas por género: mujeres y hombres respectivamente. En general podemos decir que tanto hombres como mujeres demostraron poseer una visión pro-ambientalista en la totalidad de los ítems analizados.

Dentro de los ítems analizados, destacan notablemente por la visión que muestran los estudiantes, lo siguiente: unificando TA y PA observamos que alrededor del $90 \%$ de ellos, de ambos géneros, piensan que pronto experimentaremos catástrofes ecológicas significativas debido al deterioro de la calidad de nuestro ambiente. A su vez, alrededor del $90 \%$ de los estudiantes encuestados, de ambos géneros, tienen la creencia de que el ser humano produce consecuencias desastrosas cuando interfiere con la naturaleza.

Sus respuestas para el ítem 5 son congruentes con respecto a los dos anteriores, puesto que un alto porcentaje de estudiantes de ambos sexos piensan que nosotros abusamos gravemente del ambiente natural. Es notable percibir que los jóvenes de este grupo etario y nivel educacional, ya piensan con claridad que nosotros gozaríamos de abundancia de recursos naturales si aprendiéramos a cuidarlos y a producirlos eficazmente.

Llama la atención la actitud de los jóvenes con respecto a los ítems 2 y 12 pertenecientes a la hipótesis de antroponcentrismo, donde un alto porcentaje de jóvenes manifiesta que el ser humano está destinado a gobernar la naturaleza y adecuarla a sus necesidades. Por otro lado, un alto porcentaje de estudiantes ya manifiesta la creencia de que el equilibrio de la naturaleza es sumamente frágil.

A modo de conclusión, es interesante destacar que los estudiantes chilenos de este grupo de edades, presentan, en general, una visión proambientalista tanto en hombres como en mujeres. Corresponderá a los educadores fortalecer dichas visiones y darles un mayor sustento o fundamento científico. Debemos, lo antes posible, buscar los mecanismos psico-educacionales para que dichas creencias, actitudes y percepciones, se traduzcan en comportamientos ambientales pertinentes de acuerdo a los tiempos que nos ha tocado vivir.

Debemos aprovechar eficazmente la presente década que se inicia dedicada a la Educación para la Sustentabilidad convocada por las Naciones Unidas, para crear las diversas instancias educativas, y en los diversos contextos, donde la generación en formación y la comunidad en general, puedan adquirir la alfabetización científica y tecnológica apropiada para revertir la grave situación por la cual pasa nuestro planeta en el ámbito ambiental. 
Tabla $N^{\circ} 1$. Distribución Porcentual de Respuestas para los 15 ítems NEP-Revisados

Tú estás de acuerdo o en desacuerdo con respecto a la visión ecológica de las siguientes afirmaciones:

\begin{tabular}{|c|c|c|c|c|c|}
\hline Mujeres. $\mathrm{N}=119$ & $\mathrm{TA}$ & $\mathrm{PA}$ & I & $\mathrm{CD}$ & TD \\
\hline $\begin{array}{l}\text { 1. En relación al número de habitantes que el } \\
\text { planeta posee, nos estamos acercando a un } \\
\text { tamaño límite que él puede soportar }\end{array}$ & 15,12 & 25,22 & 36,13 & 10,92 & 12,61 \\
\hline $\begin{array}{l}\text { 2. Los seres humanos tienen el derecho de mo- } \\
\text { dificar el ambiente natural para adecuarlo a } \\
\text { sus necesidades }\end{array}$ & 29,51 & 26,22 & 9,84 & 10,66 & 23,77 \\
\hline $\begin{array}{l}\text { 3. Cuando los seres humanos interferimos con } \\
\text { la naturaleza, a menudo producimos conse- } \\
\text { cuencias desastrosas }\end{array}$ & 69,17 & 19,17 & 3,33 & 3,33 & 5,00 \\
\hline $\begin{array}{l}\text { 4. El ingenio del ser humano nos asegura que } \\
\text { haremos de la Tierra un lugar apto para la vida }\end{array}$ & 10,08 & 4,20 & 18,49 & 18,49 & 48,72 \\
\hline $\begin{array}{l}\text { 5. Los seres humanos están abusando gravemen- } \\
\text { te del ambiente natural }\end{array}$ & 67,5 & 20,83 & 2,5 & 4,17 & 5,00 \\
\hline $\begin{array}{l}\text { 6. El planeta Tierra gozaría de una gran abun- } \\
\text { dancia de recursos naturales si nosotros apren- } \\
\text { diéramos a cuidarlos y desarrollarlos }\end{array}$ & 92,44 & 7,56 & 0 & 0 & 0 \\
\hline $\begin{array}{l}\text { 7. Las plantas y animales tienen tanto derecho } \\
\text { como los seres humanos a existir }\end{array}$ & 91,6 & 5,04 & 1,68 & 0 & 1,68 \\
\hline $\begin{array}{l}\text { 8. El equilibrio de la naturaleza es lo suficiente- } \\
\text { mente fuerte para hacer frente al impacto } \\
\text { ambiental generado por las naciones indus- } \\
\text { triales }\end{array}$ & 7,5 & 13,3 & 19,17 & 14,17 & 45,83 \\
\hline $\begin{array}{l}\text { 9. A pesar de todas nuestras capacidades, los } \\
\text { seres humanos aún estamos sujetos a las le- } \\
\text { yes de la naturaleza }\end{array}$ & 49,16 & 25 & 11,67 & 2,5 & 11,67 \\
\hline $\begin{array}{l}\text { 10. La denominada "crisis ecológica" que enfren- } \\
\text { ta la humanidad, es una exageración }\end{array}$ & 10,83 & 15,83 & 8,33 & 14,18 & 50,83 \\
\hline $\begin{array}{l}\text { 11. La Tierra es como una nave espacial con com- } \\
\text { partimientos y recursos limitados }\end{array}$ & 47,9 & 26,05 & 13,45 & 5,88 & 6,72 \\
\hline $\begin{array}{l}\text { 12. Los seres humanos estamos destinados a go- } \\
\text { bernar al resto de la naturaleza }\end{array}$ & 19,33 & 16,81 & 10,08 & 14,28 & 39,5 \\
\hline $\begin{array}{l}\text { 13. El equilibrio de la naturaleza es muy delica- } \\
\text { do y fácilmente alterable }\end{array}$ & 63,03 & 20,17 & 10,92 & 2,52 & 3,36 \\
\hline $\begin{array}{l}\text { 14. Los seres humanos aprenderán lo suficiente } \\
\text { acerca de cómo trabaja la naturaleza para así } \\
\text { llegar a controlarla }\end{array}$ & 22,69 & 20,17 & 21,01 & 11,76 & 24,37 \\
\hline $\begin{array}{l}\text { 15. Si nosotros continuamos deteriorando o alte- } \\
\text { rando el ambiente, pronto experimentaremos } \\
\text { una catástrofe ecológica de proporciones }\end{array}$ & 86,67 & 5,00 & 5,00 & 0,83 & 2,50 \\
\hline
\end{tabular}

Siglas:

TA: Totalmente de acuerdo

PA: Parcialmente de acuerdo

I: Inseguro

CD: Casi en desacuerdo

TD: Totalmente en desacuerdo 
Tabla N². Distribución Porcentual de Respuestas para los 15 ítems NEP-Revisados

Tú estás de acuerdo o en desacuerdo con respecto a la visión ecológica de las siguientes afirmaciones:

\begin{tabular}{|c|c|c|c|c|c|}
\hline Hombres. $\mathrm{N}=137$ & $\mathrm{TA}$ & PA & I & $\mathrm{CD}$ & TD \\
\hline $\begin{array}{l}\text { 1. En relación al número de habitantes que el } \\
\text { planeta posee, nos estamos acercando a un } \\
\text { tamaño límite que el puede soportar }\end{array}$ & 24,09 & 31,38 & 15,33 & 16,79 & 12,41 \\
\hline $\begin{array}{l}\text { 2. Los seres humanos tienen el derecho de mo- } \\
\text { dificar el ambiente natural para adecuarlo a } \\
\text { sus necesidades }\end{array}$ & 27,00 & 24,09 & 5,84 & 16,79 & 26,28 \\
\hline $\begin{array}{l}\text { 3. Cuando los seres humanos interferimos con } \\
\text { la naturaleza, a menudo producimos conse- } \\
\text { cuencias desastrosas }\end{array}$ & 62,77 & 30,66 & 1,46 & 2,92 & 2,19 \\
\hline $\begin{array}{l}\text { 4. El ingenio del ser humano nos asegura que } \\
\text { haremos de la Tierra un lugar apto para la vida }\end{array}$ & 4,38 & 3,65 & 12,41 & 27,74 & 51,82 \\
\hline $\begin{array}{l}\text { 5. Los seres humanos están abusando gravemen- } \\
\text { te del ambiente natural }\end{array}$ & 73,72 & 20,44 & 2,19 & 0 & 3,65 \\
\hline $\begin{array}{l}\text { 6. El planeta Tierra gozaría de una gran abun- } \\
\text { dancia de recursos naturales si nosotros apren- } \\
\text { diéramos a cuidarlos y desarrollarlos }\end{array}$ & 83,94 & 13,14 & 1,46 & 0,73 & 0,73 \\
\hline $\begin{array}{l}\text { 7. Las plantas y animales tienen tanto derecho } \\
\text { como los seres humanos a existir }\end{array}$ & 83,21 & 10,22 & 5,11 & 0 & 1,46 \\
\hline $\begin{array}{l}\text { 8. El equilibrio de la naturaleza es lo suficien- } \\
\text { temente fuerte para hacer frente al impacto } \\
\text { ambiental generado por las naciones indus- } \\
\text { triales }\end{array}$ & 5,84 & 12,41 & 10,22 & 19,71 & 51,82 \\
\hline $\begin{array}{l}\text { 9. A pesar de todas nuestras capacidades, los } \\
\text { seres humanos aún estamos sujetos a las le- } \\
\text { yes de la naturaleza }\end{array}$ & 53,28 & 25,55 & 7,3 & 8,76 & 5,11 \\
\hline $\begin{array}{l}\text { 10. La denominada "crisis ecológica" que enfren- } \\
\text { ta la humanidad, es una exageración. }\end{array}$ & 12,41 & 13,14 & 12,41 & 14,6 & 47,44 \\
\hline $\begin{array}{l}\text { 11. La Tierra es como una nave espacial con com- } \\
\text { partimientos y recursos limitados }\end{array}$ & 56,63 & 25 & 3,65 & 7,36 & 7,36 \\
\hline $\begin{array}{l}\text { 12. Los seres humanos estamos destinados a go- } \\
\text { bernar al resto de la naturaleza }\end{array}$ & 16,79 & 13,87 & 7,3 & 16,79 & 45,25 \\
\hline $\begin{array}{l}\text { 13. El equilibrio de la naturaleza es muy delica- } \\
\text { do y fácilmente alterable }\end{array}$ & 71,31 & 19,12 & 3,68 & 2,21 & 3,68 \\
\hline $\begin{array}{l}\text { 14. Los seres humanos aprenderán lo suficiente } \\
\text { acerca de cómo trabaja la naturaleza para así } \\
\text { llegar a controlarla }\end{array}$ & 20,44 & 21,17 & 20,44 & 14,6 & 23,35 \\
\hline $\begin{array}{l}\text { 15. Si nosotros continuamos deteriorando o alte- } \\
\text { rando el ambiente, pronto experimentaremos } \\
\text { una catástrofe ecológica de proporciones }\end{array}$ & 83,94 & 10,95 & 2,19 & 1,46 & 1,46 \\
\hline
\end{tabular}

Siglas:

TA: Totalmente de acuerdo

PA: Parcialmente de acuerdo

I: Inseguro

CD: Casi en desacuerdo

TD: Totalmente en desacuerdo 


\section{Referencias Bibliografías consultadas}

Stern, P. Dictz, T \& Guagnano, G. 1995. The New Ecological Paradigm in Social-Psychological Context. Environment and Behavior, Vol 27, 723-743.

Dunlap, R. Van Lierc, K. Mertig, A. Emmet, J. 2000. Measurin Endorsement of the New Ecological Paradigm: A Revised NEP Scale-Statistical Data Included. Journal of Social Issucs.

Kaiser, F. Wolfing, S y Fuhrer, U. 1999. Environmental Attitude and Ecological Behaviour. Journal of Environemntal Psychology 19, 1-19.

Documento Politica Nacional de Educación Para el Desarrollo Sustentablc. Borrador $\mathrm{N}^{\circ} 4$ del 31 de enero de 2006. http:// www.conama.cl
Casales, JC. 1989. Psicología Social. Contribución a su estudio. Editorial de Ciencias Sociales, La Habana.

Febles, M. 2001. Hacia un enfoque holistico del Medio Ambiente desde la psicología ambiental. Facultad de Psicología. Universidad de la Habana.

Nieto Carabeo, LM. ¿Por qué no/sí actuamos ambientalmente? La brecha entre la mente, la emoción y la conducta. http://ambiental.uaslp. mx/does/LMNCApo30306.pdf.

Schwartz, S.H (1977). Normative influences on altruism. In L. Berkowitz (ed.), Advances in experimental social psychology (Vol. 10, pp. 221-279). New York: Academic Press. 